\title{
An ontology for organizational functions: the recursive self-maintenance mechanism of the enterprise
}

\author{
David Aveiro $^{1}$, José Tribolet ${ }^{1,2}$ \\ ${ }^{1}$ Organizational Engineering Center, INESC. \\ ${ }^{2}$ Department of Information Systems and Computer Science, \\ Instituto Superior Técnico, \\ Technical University of Lisbon \\ \{david.aveiro,jose.tribolet\}@ceo.inesc.pt
}

\begin{abstract}
Although there have been great advances in business process modeling as a means to better understand and control organizations and improve the development of information systems, the existence of functions - like IT, logistics, financial, etc. - is an inescapable reality in organizations, that strongly influences aspects like change processes, or the formation of organizational units, even if a culture of "process orientation" is established. This paper tries to clarify the concept of organizational function, discusses certain organizational artifacts that seem to be of a functional nature and proposes an ontology for the purpose of allowing a consistent and coherent modeling of this recursive self-maintenance mechanism of organizations.
\end{abstract}

\section{Introduction}

The traditional method to organize and characterize an organization is through its subdivision in a hierarchy, a decomposition of units (departments, sections, teams) responsible for the execution of certain functions. This kind of representation is normally called vertical or functional [1]. What an organization does, that is, its activities and its processes, by nature, are not restricted to one function but cross horizontally all the organization, intersecting multiple departments. An example is the process of developing of a new product, that affects several departments [2].

Although the focus on processes is essential, and there has been a big emphasis on this subject, the reality is that functions continue to exist. A proof of this fact is the tendency of looking at organizations and to structure them as a matrix [3] [4] [5], where the horizontal perspectives - processes and/or projects - and vertical - hierarchy and authority in specialized knowledge - exist at the same time.

This reflects the fact that, for organizations with a certain dimension, it's impossible to have total knowledge of all details of each process (almost all of them important) that contribute in some way to their execution and outcome. There is a need to develop and maintain considerable amount of knowledge so that organizations can maintain and/or improve their performance and lower transaction costs [6].

A focus on the client, is necessary and important - so that the island phenomenon in between processes does not occur - but specialization and coordination of knowledge in functions is also necessary, so that general performance can be optimized.

As there's a need for a systematic and thorough representation of business processes and this has shown its benefits, there may also be a need of representing functions in a more systematic and thorough way, beyond the simplistic view of hierarchical diagrams and vertical representation.

One motivation for researching this potential need is that, because functions are structured to optimize performance [7], it is possible that patterns of best practices in functional behavior can be systematized (explicitly modeled) and allow knowledge capture and dissemination like, for example, transposition of best practices between different organizational functions.

Another motivation for this research is the opportunity to clarify how hierarchy relates to functional responsibilities in the processes of an organization, that is, to identify patterns and mechanisms in the circuits of authority and knowledge that assure the well functioning of an organization. We argue that this can be done by modeling in a systematic way, how the processes' performance is monitored, maintained and reported in the chains of authority and responsibility of human actors in an organization.

The concept of organizational function is scarcely defined in literature and the word function is used in 
several kinds of contexts with different meanings. This paper tries to clarify the concept of organizational function, discusses certain organizational artifacts that seem to be of a functional nature and proposes an ontology for the purpose of allowing a consistent and coherent modeling of this recursive self-maintenance mechanism of organizations.

Section 2 starts with a brief discussion on the emergence of organizations - subsection $2.1-$ continues, with a presentation of current perspectives on the function concept, in subsection 2.2 from the management field, in 2.3, from the biology field and in 2.4 from philosophy of biology, and finishes with a summary in 2.5. In section 3 we point out some organizational artifacts taken from literature, that seem to be of a functional nature: in subsection 3.1 we cover goals; in 3.2 business rules; and in 3.3 exceptions and error dynamics. In section 3.4 we present a synthesis of the several perspectives and insights found in literature, which results in an ontology for the organizational function concept. Conclusions and future work based on our findings can be found in section 4 .

\section{Function concept: perspectives}

Before addressing perspectives on the function concept we'll convey a little of the context where organizational functions emerge: the emergence of organizations themselves.

\subsection{Emergence of Organizations}

In literature, one can find two complementary views of why organizations emerge: transaction cost economics theory [8] and knowledge-based theory [6].

"The basic business unit prior to the 1840 s was the small, family-run enterprise, highly specialized, but without managerial hierarchies and no integration of business functions or areas into one firm"'[9].

"The move from traditional enterprises that used the market to coordinate owner-directed production processes to firms that employed professional managers and administrative coordination occurred with the advent of steam and coal power, rail travel, and the telegraph, that opened new markets, but more significantly, they created the possibility for new production technologies with an enormous potential for increased throughput" [6].

"The important economies of scale and distribution were not those of size but of speed. To utilize new technologies to their full required the absorption and maintenance of a large amount of new knowledge. Advances in coordination emerged along with a move toward administrative specialization through a hierarchy operated by full-time salaried managers. In this way, a vehicle was created to retain these specialized memories of production, distribution, and procurement as well as to coordinate their eventual enactment as work routines. This vehicle was the firm, with its hierarchical control of employees by managers who identified with the firm"[9].

Summarizing, one can infer that organizations emerge due to the existence of a group of persons that work together (identified with the group) and create economies of scale (that allow the lowering of transaction costs) through subordination and distribution of knowledge whose application in routines of work must be coordinated in such a way the organization survives and evolves in its mission as a whole.

A key point for understanding function dynamics in the emergence of organizations is the necessity of specialization of knowledge and of its distribution and enactment on the organization's activities and its coordination. This issue will revisited and cleared on the synthesis on section 3.4 of this paper.

\subsection{Management perspectives}

In Armstrong's perspective, an organization, while emerging, has four primary functions: (1) marketing (advertising, public relations, sales) makes people aware of the company's product or service and gets them to buy it; (2) finance (investment acquisition, accounting, strategic investments) provides initial cash flow via stock (equity) and loans, manages ongoing cash flow resulting from sales, salaries, and purchases and manages company's assets so to provide best possible return; (3) technology (development, production, delivery) develops the product or service, builds it, and delivers it to customers; (4) organization (administration, human resources, management) acquires personnel, provides direction and guidance, and provides them with what they need to be successful [10]. Other authors share similar views [5] [11] although several differences or emphasis can be found on how to separate and characterize organizational functions.

An organization can be studied from a functional or from a constructional perspective. "Taking the functional perspective means that one looks at the function of an organization with respect to its environment, and at the behavior it displays when functioning. For example, a hospital is an organization of which the (primary) function is to provide health care to people. Its behavior can be described in terms of the number of patients that are treated per period, the distribution of the treatment duration etc. A model of an organization from the functional perspective is called a black-box model" [12].

In [11] we find the organizational control systems modeling formalism (OCSM) - depicted in Figure 1 with a notion of organizational function which distinguishes two complementary aspects: (1) the goal that the function pursues; (2) the processes that realize the function based on an organizational unit. Kampfner 
considers organizations as adaptive systems and describes their structural features in terms of three binary relations on the set of functional subsystems: (1) subsystem relation, that relates any functional subsystem to its parent system (for ex. sales function considered as a subsystem of marketing function); (2) controls relation, that relates a (unique) control subsystem of a system to the operational subsystems it controls - its operational siblings (for ex. marketing's control subsystem controls operational aspects of sales); finally, (3) the reports-to relation, relates two control subsystems at contiguous levels in the control hierarchy, where the lower-level subsystem reports-to the higherlevel one (for ex. sales function's control subsystem reports-to marketing's control subsystem)[11]. The reports-to relation has a passive (sensor) nature of providing the means of monitoring of the sub-system, while the control relation has an active (actuator) nature of interference in the sub-system to change it's behavior.

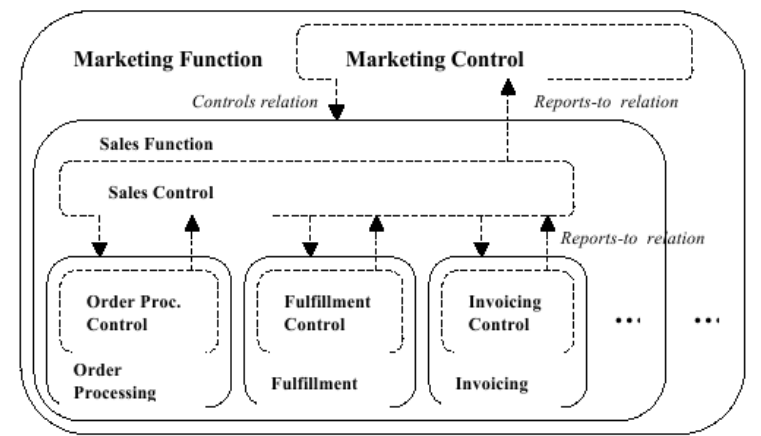

Figure 1: Organizational control systems modeling formalism

\subsection{Biology perspectives}

Smolyaninov refers to the general biological concept of "unity of structure and function" in biological systems that "exists at all levels of their organizationcell, tissue, organ and organism" and introduces the concept of equipment: "the structure is equipped with function, the function is equipped with control" which has the purpose of "overcoming the superfluous freedom of structural and functional organization" [13].

Chauvet introduces the concept of non-locality that is deduced from a new concept for biological systems, the functional interaction. The two following constraints: (1) continuous representation of state variables and (2) hierarchy of the system, result in nonlocality, i.e., a space property according to which the system depends on mechanisms that are located elsewhere in the space [14].

On the emergence of function Bickhard states that "a contribution to the maintenance of the far-fromequilibrium conditions of a far-from-equilibrium system is functional, it serves a function, for the stability, the persistence, of that system" [15].

Christensen further considers the concept of function of a normative nature while stating that "functions are essentially relations, and these process interdependency relations are what determine the nature of organisms as viable (cohesive) systems. Individual parts and processes serve normative functions within autonomous systems because of the way they satisfy the requirements of other processes within the system" [16].

\subsection{Philosophy of biology perspectives}

An important concept introduced by Christensen is of the norm. "A normative biological function is specifically a matter of traits doing what they are supposed to do. Fundamentally, a norm is an evaluative standard or principle. That is, it must be possible that there are departures from the norm, and whether there is departure or not must have value from some perspective. Resiliency is the way the system responds to potentially disruptive perturbation on the maintenance of the normal functioning" [16].

Normativity, according to Bickhard "inherently involves an asymmetric distinction between the normatively good and the normatively bad. In the case of function, this is the distinction between function and dysfunction. The asymmetry of functional/dysfunctional is derived in this model from the fundamental physical asymmetry between far-from equilibrium and equilibrium systems, in the field of thermodynamics" [15].

Katz raises also thermodynamics (negative entropy) and self-maintenance properties to characterize organizations. "the law of negative entropy states that systems survive and maintain their characteristics internal order only so long as they import from the environment more energy than they expend in the process of transformation and exportation" [17].

Wouters argues that there are at least four different ways in which the term 'function' is used in connection with the study of living organisms, namely (1) function as activity - what an organism, part, organ or substance does or is capable of doing; (2) function as causal role - the role of a part, organ, substance or behaviour of an organism in maintaining a complex activity or capacity; (3) function as survival value - the survival value of a certain part, organ, substance, or behaviour; or of a part, organ, substance or behaviour having a certain character; (4) function as selected effect - the effects for which a certain trait was selected in the past and that explain its current presence in the population [18].

\subsection{Summary}

As we can see, the essence of the organizational function concept is not clear. We can however infer one 
pattern: this word - function - is used to describe the aggregation of several processes into one more abstract macro-process, usually executed by an organizational unit. So what's the added value of calling this macroprocess a function? What properties emerge in a process that groups other processes, that justify to call it a function?

To find what these properties could be, we analyzed perspectives on the function concept from biology and philosophy of biology and find that some seem to apply to organizations, such as: control, non-locality, contribution to the maintenance of the system, interdependence relations between processes, dysfunction, normativity, resiliency, survival and selection.

In the next section we cover some artifacts of organizations, that seem to be of a functional nature due to the apparent exhibiting of some of the previously referred properties.

\section{Functional artifacts in organizations}

\subsection{Goals}

Quite a lot has been written on strategy in organizations and its specification in interconnected goals. Kavakli presents in her work, a comprehensive list and analysis of current methods: in respect to goals, "several classification axes have been proposed in the literature. Functional goals underlie services that a business process expected to deliver whereas nonfunctional goals refer to expected process qualities such as security, safety, performance, usability, flexibility, customizability, interoperability, and so forth. This typology is overly general and can be specialized. Performance goals are specialized into time and space performance goals, the former being specialized into response time and throughput goals" [19].

Kueng and Kawalek simply put it: "goals are statements which declare what have to be achieved or even avoided by a business process" [20].

As we saw before (section 2.3), the interrelations between processes, in terms of requirements is a functional artifact. Such requirements state what is required for a process to satisfy and can also state what is to be avoided. We can see a clear parallel with the function/dysfunction asymmetry pointed in section 2.4 which is a consequence of a a departure or maintenance of the processes norm. This means that one can consider the goal artifact - commonly used in management and enterprise modeling - as an expression of the functional property or concept of norm.

\subsection{Business rules}

A business rule is a statement that defines or constrains some aspect of the business. It is intended to assert business structure or to control or influence the behavior of the business [21].

According to Eriksson and Penker, "business processes are governed by rules (business rules). Rules define restrictions that are imposed to the activities, indicating how the business should function. Rules can be built due to external legal issues, or as an internal mechanism to guarantee that goals are achieved" [22].

From these statements and taking into account the insights presented in previous sections, what is commonly called as business rules appear to be functional restrictions because they make processes comply with requirements from other processes, internal or external to the organization.

While discussing about function and dysfunction, Christensen presents the concept of resiliency as a functional artifact, defining it as "the way the system responds to potentially disruptive perturbation on the maintenance of the normal functioning" [16].

We infer that business rules can be considered as resiliency mechanisms that act, internally in, or externally to, a process, to maintain a norm of functioning.

One important question that arises, to understand function dynamics in an organization is: how business rules (resiliency mechanisms) are established? This subject is addressed in the following section.

\subsection{Exceptions and error dynamics}

As it was seen in section 2.4, if there is a departure from a norm in a process, it enters in a dysfunctional state. In the previous section we already understood which artifacts are used to try to stop and prevent dysfunction, but what provokes these departures?

As we saw also previously, every process requires outputs from other processes in certain ranges of variations (inside the norm). Mourão and Antunes discuss in their work that "unexpected exceptions correspond to unpredicted situations for which the system can not suggest any solutions" [23]. They are referring to the workflow category of systems, but we adopt the same concept for an organization as a system.

When no current known resilience mechanisms (current business rules) are able to cope with the exceptions, that an organization is facing (unexpected variations in inputs/outputs of processes), it enters in a dynamic, unstable and eventually very complex context where new resilience mechanisms have to be established to prevent or cease dysfunction, for the survival (maintenance) of the organization.

Bickhard addresses in his work, the subject of error dynamics which can lead to the construction of "error vicariants" which is another name for resilience mechanisms. An important contribution from this 
author is the elicitation of interactive error and construction error.

"It will be beneficial to a recursively selfmaintenant system - it will increase its adaptiveness to develop or evolve dynamics of interaction with the environment whose primary function is to contribute toward the regulation of other interactions. In particular, insofar as the informational redundancies of the environment permit, it will be beneficial to develop interaction forms that can serve as surrogates or vicariants for dynamic errors. Vision is a modality of interaction, for example, that is largely dedicated to serving such error vicariant functions. It is much better to encounter the visual error of approaching a wall on your way to the next room than it is to actually bump into that wall: the visual interaction is a surrogate for the collision. The comparison of a visual encounter with a cliff and a physical encounter with a cliff is even more dramatic"[24].

In organizational terms, we can infer that these interactive errors constitute the triggers or conditions embedded in business rules, that will guide the execution of business processes.

The notion of constructive error arises from what Bickhard defines as microgenesis: "a form of construction - a micro-construction. Constructivism is usually thought of in connection with learning and development: new learning or development is constructed - rather than, for example, being passively impressed by the environment into the mind, such as if the mind were a waxed slate. The basic intuition of the necessary intimate relationship between microgenesis, on the one hand, and the constructions of learning and development, on the other, is that the stabilized setting up of old and successful dynamics (microgenesis) must occur in the same process as the variational setting up of new trial dynamics (heuristic learning, development, problem solving) in order for the constructive 'location' of the successful constructions to be available to heuristically guide the microgenetic, microconstructive, processes of new trial constructions" [24].

Simplifying, trial solutions to new problems must be in some sense near to well-established solutions to old problems - to which the new problems are similar, and new solutions are micro-constructed, based on similar patterns of old successful dynamics. Microgenesis and error dynamics (and meta-dynamics) are quite complex to be further addressed here, but they provide a powerful paradigm that can be used to explain in a systematic and thorough way the mechanisms and patterns of processes inherent to the construction of new resilience mechanisms, that will guide business operation and anticipate or react to previously unknown exceptions in the organization's activity.

\subsection{Synthesis: an ontology for organizational functions}

We recall that the purpose of this paper is to clarify the organizational function concept, while proposing an ontology for its coherent modeling. This purpose comes in the context of research work done at Centro de Engenharia Organizacional ${ }^{1}$ (CEO) of INESC INOV, whose current view of organizational modeling is synthesized in recent work of Sousa et al. [25] In this work, key concepts for modeling the organization's enterprise architecture using the Unified Modeling Language (UML) are discussed: "Enterprise architecture consists on defining and understanding the different elements that shape the organization and how these elements are inter-related with the purpose of understand and facilitate organizational evolution and change. It separates core organizational concerns as different architectural views".

The authors argue that "modeling the multidimensional aspects of the enterprise should be organized into five architectural components: Organization, Business, Information, Application and Technological architectures. These five components are supported in a small set of fundamental concepts described using UML 2.0. Any organization model may be abstracted to three elements: Activity, Role and Entity".

It is our purpose to complement the above mentioned work, incorporating the functional dimension (or architecture) of the enterprise, being this paper a first step in that direction.

An ontology is a specification of the objects, concepts and other entities that are assumed to exist in some area of interest and the relationships that hold among them.

Process - The area of interest is organizational functions, in the context of enterprise architecture modeling, and we propose an ontology for the organizational function concept, anchored on the fundamental concept of activity. An activity is an abstraction representing how a number of entities collaborate through roles in order to produce a specific outcome. Similarly to an algorithm, an activity aims accomplishing some task which, given an initial state, will always end in finite time and in a recognizable endstate. An activity may also be functionally decomposed into a finite set of further activities, thus add detail to the specification. To maintain coherence with the cited work on biology and philosophy fields, in this paper we use the equivalent name: process. A forthcoming paper [26] proposes a modeling framework, elicited from this ontology and coherently based on the previously referred fundamental concepts (activity, role and entity).

1 information about this research centre is available at http://ceo.inesc.pt 
Function - This word has its root in the latin word "functio" which means functioning, which in turn means "doing well in a regular way". Functions are process interdependency relations that determine the nature of organizations as viable (cohesive) systems. Processes in an organization are all interrelated or interdependent in some way due to the intertwined nature of its operation. Changes in the characteristics of inputs or outputs of processes in an organization will affect in some way (dysfunctional or not) the operation of other processes elsewhere (interdependence [16] and non-locality [14]).

Norm \& Resilience - So that the organization can survive (maintain a capability of recursive self maintenance [24]) certain processes (or parts of processes) will have to serve the capability of maintaining the conditions on which the organization is able to maintain its cohesion and survive (primary goal of any organization). That is, an organization has to have processes (or parts of the processes themselves business rules) which are dedicated to monitor if key state variables are in the state of "doing well in a regular way" - or in other words, respecting the established norm or goal for them - and work as (or invoke elsewhere) resilience dynamics when there is a departure from the norm.

Exceptions \& Microgenesis - If in a certain process there is a departure from the norm, this can be due to: an expected exception - the case in which previously existing business rules (methods embedded in the process) or other processes (invoked due to the exception) will work as a resilience mechanism to try to restore the norm; or an unexpected exception - the case where the organization will enter in a dynamic space where microgenesis processes will occur. These microgenesis processes can be highly complex and unpredictable, depending on the degree of complexity of the problem that caused the exception and amount of knowledge necessary to investigate and understand the aspects of the problem and heuristically (through trial and error tentatives, based on similar situations and its results analysis) construct or change existing processes and/or business rules to circumvent or solve the problem that caused the exception.

As we saw in section 2.1, just as the necessity of specialized knowledge (and its coordination) was at the genesis of the organization [6] [9], also specialized knowledge is at the heart of the micro construction (or change) of the organization, as a way of adaptation (realized in new resilience processes/rules) to new environmental conditions.

Summary - the ontology of an organizational function and its dynamics is depicted on Figure 2. Rectangles mean relevant entities for the dynamics. Rectangles with one side arrowed mean each context of dynamics for a certain organizational function (operation, monitoring, resilience and microgenesis). The flow between these contexts is described by the decision boxes and continuous line arrows. The dashed arrows represent the information flow in each context. The dashed boxes mean the operational facet of an organizational function, while the rest of the symbols mean the meta-plan than contains the other elements of the functional dimension. Given a process $\mathrm{X}$, we can elicit an organizational function by stating the following interrelated artifacts: (1) a norm (goal value) for a certain state variable of the process. More state variables can exist and for each of them, one or more norms can be established, each of these corresponding to an individual organizational function; (2) Which

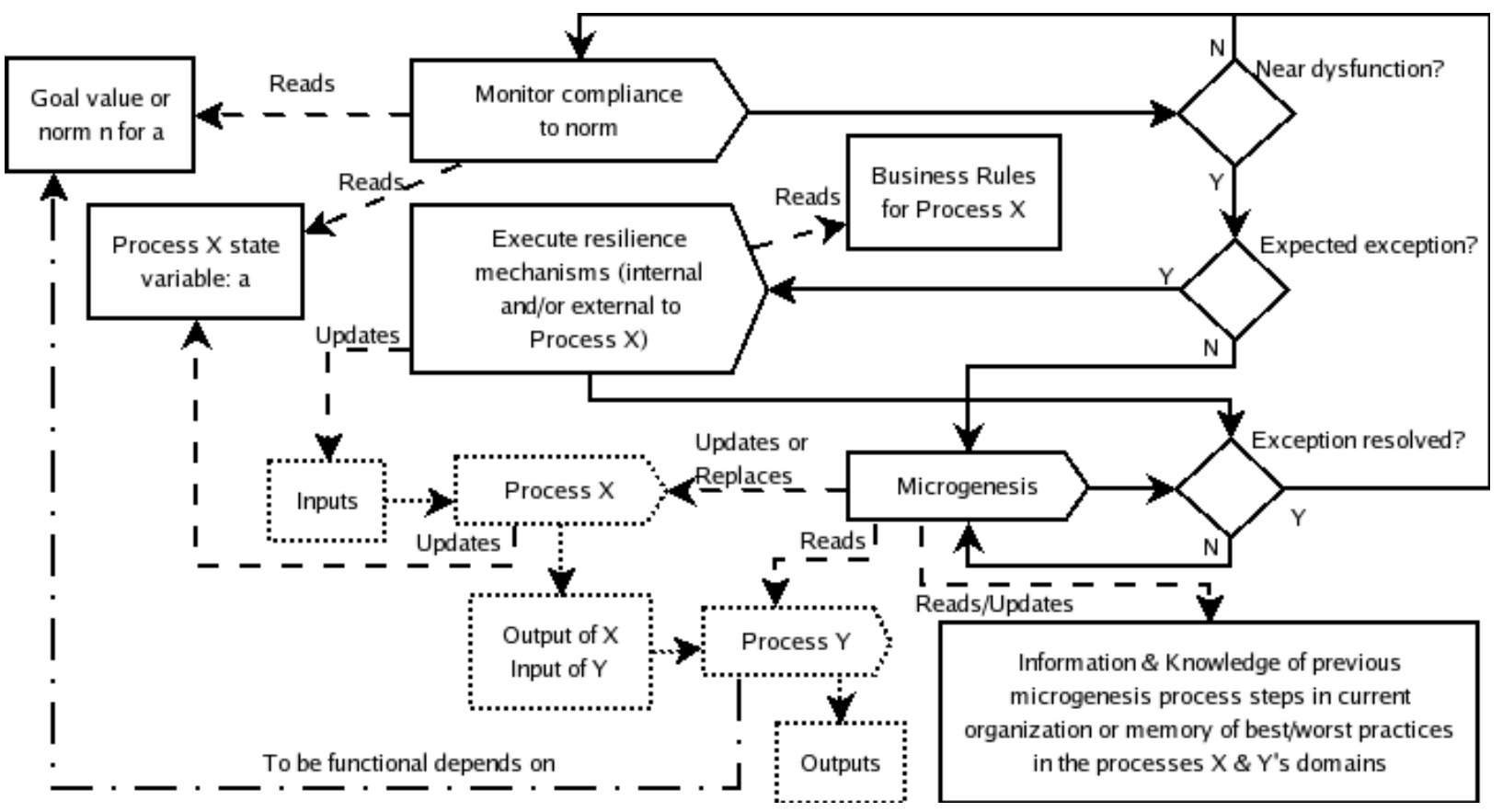

Figure 2: Depiction of an organizational function's artifacts and dynamics 
other process $\mathrm{Y}$ (or processes) depend on this norm, in order to remain functional; (3) The set of business rules (embedded in the process itself, or other processes) that work as resilience mechanisms to expected exceptions and try to reestablish the norm to the process functioning, either by internal measures, or by invocation of other processes that will make inputs of process $X$ change in a way it returns to normal functioning (4) Set of specialized knowledge related to process $\mathrm{X}$ and $\mathrm{Y}$ 's domain, that is, information about business rules that: (i) worked or not in previous real (microgenesis) situations in the organization (ii) are best (or not so good or even bad) practices from theoretical (or real) situations accumulated in the memory of the agents executing the microgenesis processes. This knowledge is valuable input for the microgenesis process of treating unexpected exceptions, that will select new successful business rules to be integrated in process $\mathrm{X}$, or ultimately replace it. During a microgenesis process, not only $\mathrm{X}^{\prime} \mathrm{s}$ resilience business rules, but all entities of $X^{\prime}$ 's organizational function may be refined or even replaced.

These artifacts are grounded philosophically in a solid manner, thanks to the clear parallel found with Wouters' four notions of function [18]: (1) as an activity - the process it self - process X; (2) as causal role - the process or processes that depend on the process - process Y; (3) as survival value - goal value for a certain part of the process and resilience mechanisms that try to maintain its norm - X's state variable and norm that restricts it and the business rules that realize these restrictions; (4) as selected effect - the selected resilience mechanisms in the end of a microgenesis process that started due to an unexpected exception - collected microgenesis information, which is, at the same time, input and output of the microgenesis process.

Organizational functions are the mechanism of selfmaintenance of organizations and this mechanism is recursive [24], because the process (or processes) that constitute a resilience mechanism, may themselves get dysfunctional and need to have their own organizational functions elicited for systematic invocation of other resilience mechanisms, typically involving horizontal or vertical escalation of exceptions [23], which can, in an extreme situation, lead to total reconfiguration of an organization or its disintegration due to inability to cope with the new environmental conditions.

\section{Conclusions and future work}

For the clarification of the function concept, we bridged insights from diverse areas like management, biology and philosophy of biology end elicited an ontology that describes the main artifacts of the functional dimension of an organization and their interrelation, for the purpose of modeling this concern of an enterprise architecture. To arrive at the proposed artifacts, we have assumed that, in an enterprise, the functional dimension is the one whose mission is to assure its self-maintenance.

We find that current practices of management and modeling disregard the relations between functional artifacts as systematized in our proposed ontology. In forthcoming work [26], based on the ontology hereby presented, we propose a framework that allows a separation of concerns between the modeling of operational aspects of collaborations as presented in [25] and the aspects of interdependences, monitoring and learning, inherent to the functional dimension of the enterprise. We also present an application of the ontology and framework to a theoretical business situation, as a proof of concept.

This framework can also be used as a basis to capture the dynamics of monitoring and evolution (or adaptation) of the enterprise, in face of expected or unexpected changes in its environment, thus allowing not only sustained survival, but a conscious evolution.

To model organizational functions of activities is a way to capture knowledge about resiliency solutions, that is, how resources are managed in the context of disruptive deltas in key state variables of certain processes. This knowledge would otherwise remain tacit in the minds of human actors of organizations. It's also possible to capture information on which steps made current business practices successful (in previous situations of unexpected exceptions) and thus allow a more conscious process of evolution or adaptation to new unexpected environmental perturbations. That is, information of the process of learning of an organization can be collected and reused in other learning situations.

Also, through the elicitation of networks of interdependences between processes (networks of norms), we aim to bring the possibility of (automatically or semi-automatically) detecting which processes are more vital and establish priorities for creating proactive mechanisms of resiliency that can prevent and avoid propagation of dysfunction in a timely manner.

It also allows to connect the functional artifacts with human actors, allowing the establishment of clear (traceable) chains of responsibility and authority in the functioning of an organization's processes. This is useful to discuss hierarchies and reformulate them in a manner more suitable to streamline the self-maintenance and evolution of an organization.

To further develop current work, two graduate theses are currently assigned for starting soon. One has the purpose of extending a modeling tool (System Architect, Casewise, MooD or other) to allow a coherent application of the proposed framework to model functional dependencies between processes as well as identifying networks of interdependences and elicit possible risks on vital processes, and allow 
justified proposals of new business rules (resilience mechanisms) to reduce the risk of dysfunction.

The other theses aims to create an intuitive tool for capturing in real-time, or after situations of unexpected exceptions, structured information about dynamics of selection of new business rules (microgenesis dynamics) and, at the same time, to provide informational support to actors participating in these dynamics, by allowing search of information available in accumulated logs and, if possible, automatic suggestion, based on IA techniques (like Case Based Reasoning, Rule induction algorithms of machine learning or others). Other purpose is to have this tool integrated with work being developed on the previously referred proposal.

\section{Acknowledgement}

Research work that led to results presented in this paper was possible thanks to the financial support of a PhD scholarship (Ref.: SFRH / BD / 13384 / 2003) subsidized by "Fundação para a Ciência e a Tecnologia Ministério da Ciência, Tecnologia e do Ensino Superior" of the Portuguese government and by the European Social Fund (FSE), in the scope of the "III Quadro Comunitário de Apoio".

\section{References}

[1] Kavakli V., Loucopoulos P., Goal-Driven Business Process Analysis Application in Electricity Deregulation, 1998

[2] Loucopoulos P., Kavakli E., Enterprise Modelling and the Teleological Approach to Requirements Engineering, 1995

[3] Applegate Linda M., McFarlan F.Warren and McKenney James L., Corporate Information Systems Management 5th Edition, McGraw-Hill Companies, Inc, 1999

[4] Bavel Richard van and Bronkhorst Jeroen, Embedding and managing IT processes in an organization, The Guide to IT Service Management Volume I, Addison-Wesley, Hewlett Packard, Amstelveen, the Netherlands, 2002

[5] Sutcliffe Norma G., S 483 Information Systems Management, Session Notes, DePaul University, 2004

[6] Weeks J. and Galunic C., A theory of the cultural evolution of the firm: the intra-organizational ecology of memes, 2003

[7] V. Kavakli, P. Loucopoulos, Goal-Driven Business ProcessAnalysis Application in Electricity Deregulation, 1998

[8] Coase R. H., The Nature of the Firm, 1937

[9] Chandler Alfred D., The Visible Hand: The Managerial Revolution in American Business, Belknap Press, 1980

[10] Armstrong E., The Tetrahedral Model of a Business, 2000
[11] Kampfner R., Adaptive systems view of organizational functions, Proceedings of the 45th Annual Conference ofThe International Society for the Systems Sciences, College of Engineering and Computer Science, The University of Michigan-Dearborn, 2001

[12] Dietz J.L.G., Generic recurrent patterns in business processes, Business Process Management: International Conference, Bpm 2003, Eindhoven, the Netherlands, 2003

[13] Smolyaninov V. V., Structure, function, control: a system-constructive approach, Laboratory of Biomechanical Systems, Russian Academy of Sciences, 1998

[14] Chauvet G. A., Non-locality in biological systems results from hierarchy. Application to the nervous system., Institut de Biologie Theorique, Universite d'Angers, 1993

[15] Bickhard Mark H., Process and Emergence: Normative Function and Representation, Department of Philosophy, Lehigh University, 2004

[16] Christensen W. D. and Bickhard M. H., The process dynamics of normative function (The Philosophy of Biology), Monist, Hegeler Institute, 2002

[17] Katz Daniel and Kahn Robert L., The Social Psychology of Organizations, The Social Psychology of Organizations, 1978

[18] Wouters Arno G., Explanation Without A Cause, Ph.D. thesis, Utrecht University, The Netherlands, 1999

[19] Kavakli E., Modeling organizational goals: Analysis of current methods, Proceedings of the 2004 ACM symposium on Applied computing, ACM Press, 2004

[20] Kueng P., Kawalek P., Goal-Based Business Process Models: Creation and Evaluation, Informatics Process Group (IPG), Department of Computer Science, University of Manchester, UK, 1996

[21] Business Rules Group, Defining Business Rules - What Are They Really?, 2000

[22] Eriksson, H., Penker, M, Business Modeling with UML: Business Patterns at Work, OMG Press, Wiley Computer Publishing, 2000

[23] Mourão, H. and P. Antunes, A Collaborative Framework for Unexpected Exception Handling, Groupware: Design, Implementation, and Use, Heidelberg, SpringerVerlag, 2005

[24] Bickhard M. H., The Dynamic Emergence of Error Avoidance and Error Vicariants, Journal of Experimental \& Theoretical Artificial Intelligence, Taylor and Francis Ltd, 2001

[25] Sousa P., Caetano A., Vasconcelos A., Pereira C., Tribolet J., Enterprise Architecture Modeling with the Unified Modeling Language, Enterprise Modeling and Computing with UML, IRM Press, 2006

[26] Aveiro D. and Tribolet J., Organizational functions and enterprise self-maintenance: a framework for integrating modeling, monitoring and learning, 3rd International CIRP Conference on Digital Enterprise Technology, Setúbal, Portugal, September 2006 (Accepted). 